\title{
molecules
}

ISSN 1420-3049

www.mdpi.com/journal/molecules

Review

\section{Role and Regulation of Glutathione Metabolism in Plasmodium falciparum}

\section{Sylke Müller}

Institute of Infection, Immunity and Inflammation, College of Medical, Veterinary and Life Sciences, University of Glasgow, 120 University Place, Glasgow G12 8TA, UK;

E-Mail: sylke.muller@glasgow.ac.uk; Tel.: +44-(0)141-330-2383; Fax.: +44-(0)141-330-4600

Academic Editor: Angela Calderon

Received: 10 April 2015 / Accepted: 1 June 2015 / Published: 8 June 2015

\begin{abstract}
Malaria in humans is caused by one of five species of obligate intracellular protozoan parasites of the genus Plasmodium. P. falciparum causes the most severe disease and is responsible for 600,000 deaths annually, primarily in Sub-Saharan Africa. It has long been suggested that during their development, malaria parasites are exposed to environmental and metabolic stresses. One strategy to drug discovery was to increase these stresses by interfering with the parasites' antioxidant and redox systems, which may be a valuable approach to disease intervention. Plasmodium possesses two redox systems - the thioredoxin and the glutathione system-with overlapping but also distinct functions. Glutathione is the most abundant low molecular weight redox active thiol in the parasites existing primarily in its reduced form representing an excellent thiol redox buffer. This allows for an efficient maintenance of the intracellular reducing environment of the parasite cytoplasm and its organelles. This review will highlight the mechanisms that are responsible for sustaining an adequate concentration of glutathione and maintaining its redox state in Plasmodium. It will provide a summary of the functions of the tripeptide and will discuss the potential of glutathione metabolism for drug discovery against human malaria parasites.
\end{abstract}

Keywords: malaria; glutathione; drug discovery; drug target; redox metabolism; antioxidant

\section{Introduction}

Malaria in humans is caused by five species of protozoan parasites of the genus Plasmodium, namely $P$. falciparum, $P$. vivax, $P$. ovale, $P$. malariae and $P$. knowlesi (www.cdc.gov). The most 
severe form of the disease is caused by P. falciparum and about $90 \%$ of the 600,000 annual deaths are attributable to an infection with this parasite species in Sub-Saharan Africa, with children under the age of 5 and pregnant women being most vulnerable to the infection [1].

The parasites causing malaria have a complex life cycle, which depends on their sexual development in female Anopheles mosquitoes where the human infective transmission stages (the so-called sporozoites) are formed. When the mosquito bites a human, the infective sporozoites invade hepatocytes, where they undergo a first, non-symptomatic multiplication cycle by schizogony, before thousands of merozoites are released into the blood stream. Here, they infect red blood cells (RBC) and within $24 \mathrm{~h}$, $48 \mathrm{~h}$ or $72 \mathrm{~h}$ (depending on the Plasmodium species) develop into up to 32 daughter merozoites. These egress from the host cells, infect fresh $\mathrm{RBC}$ and begin a new round of multiplication. At this stage of their cycle, the parasites can also develop into gametocytes, which are infective to mosquitoes-thus closing the cycle. The RBC stages of Plasmodium cause the symptoms of malaria and are those that most of the currently available chemotherapies act against. However, resistance against a lot of these drugs rapidly develops and spreads, and this alarming situation underpins the urgency for the development of new intervention strategies to fight the parasites and thus prevent or treat malaria, especially as an efficacious vaccine is still elusive.

Evidence suggests that the geographical distribution of RBC genetic traits such as the thalassemias, sickle cell anaemia and glucose-6-phosphate dehydrogenase deficiency (G6PDH-deficiency) correlate with reduced severity of malaria [2-6]. How these haemoglobinopathies interfere with the infection remains uncertain although some hypotheses suggest increased oxidative stress may play a pivotal role. The underlying mechanisms may involve the oxidative damage to the host RBC that occurs upon infection with Plasmodium and that these are exacerbated by the genetic traits $[5,7,8]$. The phenotype of Plasmodium-infected RBC harbouring one of the genetic traits resembles that displayed by senescent or pathologically damaged RBC [5] and this may result in a reduced "Plasmodium-infectivity" [9]. An infection with Plasmodium results in an increased level of reactive oxygen species in the infected RBC [10-14]. This leads to premature oxidative damage of the infected RBC, which ultimately affects membrane composition and protein conformations of the infected host cell and enhances the generation of lipid peroxides such as 4-hydroxynonenal. These lipid peroxides negatively affect RBC membrane integrity and impact on the host's immune response in vitro and in infected individuals $[15,16]$.

Nevertheless the parasites manage to thrive in this hostile environment presumably because they possess an extraordinary capacity to withstand the oxidative challenges that they encounter during their intra-erythrocytic growth $[17,18]$. Two redox systems help the parasites in this fight for survival. They possess functional and highly elaborate thioredoxin and glutathione redox systems in addition to other necessary components of an active antioxidant defence [18-20]. Both redox systems rely on a supply of NADPH required as reducing equivalents for their disulphide oxidoreductases thioredoxin and glutathione reductase (GR), respectively [18,20,21]. Usually, NADPH is provided by the pentose phosphate shunt (PPP) and this is also the case in Plasmodium [22-25]. In P. falciparum it was found that glucose-6-phosphate dehydrogenase and 6-phosphogluconolactonase form a bifunctional enzyme providing NADPH for downstream redox reactions [26]. Although this enzyme might supply the cytoplasm with sufficient reducing equivalents, it is likely that other NADPH-generating dehydrogenases present in the parasite's organelles also contribute to the supply of NADPH. For instance, Plasmodium possess two $\mathrm{NADP}^{+}$-dependent glutamate dehydrogenases - one is cytosolic, while the other one is 
located in the plastid-like organelle called apicoplast [27,28]. In addition, the parasites contain a mitochondrial $\mathrm{NADP}^{+}$-dependent isocitrate dehydrogenase generating NADPH [29]. Thus, reducing power is also available in the apicoplast and mitochondrion-both organelles that also need to be protected against endogenous metabolic oxidative stress and therefore depend on an adequate supply of the redox co-factor. Over the past decades, the thioredoxin and glutathione redox systems have been extensively studied and their importance for parasite survival was evaluated. This review will focus on glutathione and its roles in parasite biology and it will discuss whether the pathways controlling glutathione homeostasis are suitable for drug discovery against malaria. In the following text, glutathione will be used when both reduced glutathione (GSH) and oxidized glutathione (glutathione disulphide, GSSG) are referred to.

\section{Glutathione}

The tripeptide glutathione ( $\gamma$-glutamyl-cysteinyl-glycine) represents the major thiol redox buffer in almost all eukaryotes and thus is a determinant of the intracellular redox status. GSH is a co-factor for antioxidant and detoxification enzymes such as glutathione peroxidases (GPx) and glutathione S-transferases (GST) and its abundance and redox state impacts on the cell's ability to defend itself against internal and external stresses. Glutathione normally is highly abundant (in mM concentrations) and as an enzyme co-factor and an antioxidant it is active in its reduced form as GSH, which is maintained by the NADPH-dependent GR [30]. The ratio of GSH to GSSG is usually high (>30:1) to ensure maintenance of the intracellular reducing environment. The only compartment with a more oxidising redox status is the endoplasmatic reticulum (ER), where the GSH/GSSG ratios have been reported to be up 1:1 [31]; this is necessary to ensure structural integrity of excreted or membrane proteins often containing multiple intra- or inter-molecular disulphide bonds. Therefore, GSH levels and GSH/GSSG ratio provide a suitable indicator of cellular redox homeostasis and oxidative stress [32-34].

This is also the case in the malaria parasite Plasmodium. These intracellular protozoan parasites possess a parasite-specific GR, which recycles GSSG and maintains thiol redox balance at the expense of NADPH [19,21,35-37]. The GSH/GSSG redox couple in intra-erythrocytic parasites has a highly reducing redox potential $E^{\circ}{ }_{G S H}$ of $-314 \mathrm{mV}$ comparable with the GSH/GSSG redox potential in other organisms [38]. PfGR is found in both, the cytoplasm and the apicoplast of the parasites although they apparently only possess a single gene encoding the protein. It was shown that the dual location is attributable to alternative translational initiation directing the protein into cytoplasm and plastid organelle. This mechanism of guiding proteins encoded by a single gene into different cellular locations has been adapted by Plasmodium quite efficiently and it was found that thioredoxin reductase is also dually targeted through this mechanism to both cytoplasm and mitochondrion [39].

Plasmodium also possesses the two enzymes responsible for the de novo biosynthesis of GSH, $\gamma$-glutamylcysteine synthetase ( $\gamma$-GCS) and glutathione synthetase (GS), respectively. The biosynthetic pathway for GSH establishes a constant source of the tripeptide in the parasites cytoplasm, as long as the amino acid precursors (glutamate, cysteine, and glycine) are available [40-45]. GSH biosynthesis is of particular importance in Plasmodium-infected RBC because the infected RBC "lose" or turnover glutathione rapidly and thus have to constantly replenish the tripeptide via their biosynthetic 
pathway $[40,44,46]$. It has also been suggested that uptake of GSH may contribute to sustain intracellular GSH levels; however, the significance of this source of GSH remains to be established $[44,47,48]$. The reason for the rapid glutathione turnover is unknown but one possible interpretation of this finding was provided by Ginsburg and colleagues suggesting that GSSG generated in the parasite may be excreted into the host RBC, which apparently has a decreased ability (or lost the ability altogether) to synthesise GSH de novo and this way provides the co-factor to its host cell, which contains GR to reduce GSSG back into GSH [49]. Another study provided evidence that it is primarily GSH that is excreted from the parasites [46].

Plasmodium-infected RBC encounter oxidative stress through the parasite's metabolic activity and also the host's immune system. Plasmodium incorporates and degrades the major host cell protein hemoglobin in its acidic digestive vacuole (DV) by a set of peptidases [50]. Heme (ferroprotoporphyrin IX containing $\mathrm{Fe}^{2+}$ ) and hemin/hematin (ferriprotoporphyrin IX containing $\mathrm{Fe}^{3+}$ ), highly redox active metabolic by-products of hemoglobin degradation, are released and the majority is biomineralised and sequestered in the form of hemozoin crystals within the DV to prevent downstream toxicity [51]. However, it has been suggested that appreciable amounts of heme may be released into other compartments of the parasite-infected RBC, where they interfere with protein and membrane integrity and affect the thiol redox balance [52]. One hypothesis is that hemin/hematin is removed by non-enzymatic degradation/peroxidation using GSH as observed in vitro [52,53]. This was recently confirmed and it was further demonstrated that hemin/hematin degradation was accelerated by the action of EXP1 (exported protein 1), a protein with GST activity located in the parasitophorous vacuole [54]. This enzyme activity may rely on GSH excreted from the parasites (which was demonstrated by Barrand and colleagues [46,55]) and this could be the explanation for the unusually fast turnover of the tripeptide observed in earlier studies [40,49]. In addition, the parasites possess a second, cytosolic GST, which until recently was considered to be the sole GST existing in Plasmodium. The protein shows classical GST activity using artificial substrates, is highly abundant in the parasite cytoplasm and was shown to bind and thus sequester hemin as ligandin; the enzyme also has some considerable antioxidant activity [56-60]. Curiously, the parasites contain no genuine GPx and the protein with a high sequence similarity to GPx from other organisms depends on thioredoxin as reducing cofactor [61]; further it needs to be mentioned that Plasmodium do not possess a gene encoding catalase [62]. Therefore, it is presumed that the multiple organelle-specific peroxiredoxin-linked detoxification systems of hydroperoxides provide the majority of the parasites' antioxidant capacity $[18,20,63,64]$.

Total glutathione levels have been determined in isolated $P$. falciparum and $P$. falciparum-infected RBC multiple times and depending on the method used they vary considerably between different studies and different Plasmodium species [40,41,44,45,49,65]. Total glutathione concentrations (comprising GSH and GSSG) in the range of 1 to $2 \mathrm{mM}$ were reported in Plasmodium-infected RBCs and the parasites themselves [19,38,44,45], although in some studies lower concentrations were measured [41,65-67]. These discrepancies may be attributed to differences in sample preparations as well as detection methods; it was for instance found that concentrating the parasites or parasitized RBC using Percoll or MACS columns exerts oxidative stress on the cells leading to a reduction of GSH levels [45]. There are multiple ways of determining GSH levels in Plasmodium-infected RBCs or isolated parasites: (1) HPLC-based methods using derivatisation of thiols with fluorescent probes [44]; 
(2) colorimetric spectrometric assays using Ellman's reagent [68] and (3) recently an in vivo method was developed using P. falciparum that express a human glutaredoxin 1 (hGrx) analogue linked to redox-active GFP (hGrx-roGFP), which allowed determining the GSH redox state in the parasites in real time without disrupting the parasite-host cell unit [38]. All detection methods conclusively demonstrate the GSH redox state is highly reducing in both host-parasite unit and isolated parasites, as expected, and moreover, the in vivo assay showed that the GSH/GSSG ratio and concentration is stable over time in unstressed parasites [38]. This is particularly interesting in light of the previously mentioned high turnover of GSH in the parasite suggesting an active biosynthesis of the tripeptide to compensate for its constant efflux from the parasite- be it as GSH, GS-X or GSSG [40,44,46,48]. Using hGrx-roGFP expression in Plasmodium now provides an excellent tool to monitor GSH redox balance in real time, assess the effect of pro-oxidants and other metabolic stressors and it should be possible to transfer the system to monitoring the redox balance in the different parasite organelles. This is an extremely exciting prospect and presents an excellent way to evaluate, for instance, the in vivo redox requirements of the protein trafficking machineries that are responsible for the transport of parasite proteins (virulence factors) into the host cell and its membranes operating in the Plasmodium-infected $\mathrm{RBC}$ or into the parasite's organelles such as apicoplast, mitochondrion and ER.

\section{The Functions of GSH in Plasmodium}

Plasmodium possesses a number of GSH-dependent enzyme systems including GSTs, glyoxalases and glutaredoxins as well as a GPx-like protein [19,20,54,60,69]. Thus, GSH is important for detoxification and thiol-disulphide exchange reactions, however, its impact on the antioxidant capacity of the parasites is less well-defined especially as Plasmodium GPx is more likely to use thioredoxin as reducing co-factor rather than GSH [61]. It was shown that GST1, apart from detoxifying electrophiles and acting as a ligandin for hemin, also has GSH-dependent peroxidase activity and thus is an integral part of the parasite's antioxidant machinery (Figure 1) [56,58]. A second GST activity (GST2), attributed to a protein previously called EXP1, was recently revealed and the protein is located in the parasitophorous vacuolar membrane surrounding the parasite. GST2 was shown to contribute to hemin/hematin-detoxification in a GSH-dependent manner as well as playing a role in the detoxification of the antimalarial artesunate [54]; its antioxidant capacity was not investigated. The glyoxalase system is another vital cellular component depending on a supply of reduced GSH and it was shown to be crucial for parasite survival [69]. Glyoxalases conjugate 2-oxoaldehydes such as methylglyoxal, a toxic metabolic by-product of glycolysis, to GSH and lead to the generation of non-toxic hydroxycarboxylic acids such as D-lactate, which are excreted from the cell. Apart from these pathways, the parasites possess numerous glutaredoxins and glutaredoxin-like proteins which require GSH as co-factors [70]. Together this indicates that the GSH system might not have the expected and often prominent role in providing an efficient antioxidant defence to Plasmodium but it seems to offer a powerful redox buffer that maintains intracellular redox homeostasis and is mainly involved in detoxification reactions through GSTs and glyoxalases and provides regulation of protein functions via thiol-disulphide exchange reactions as exemplified by the strong level of glutathionylation determined in Plasmodium proteins [71]. 


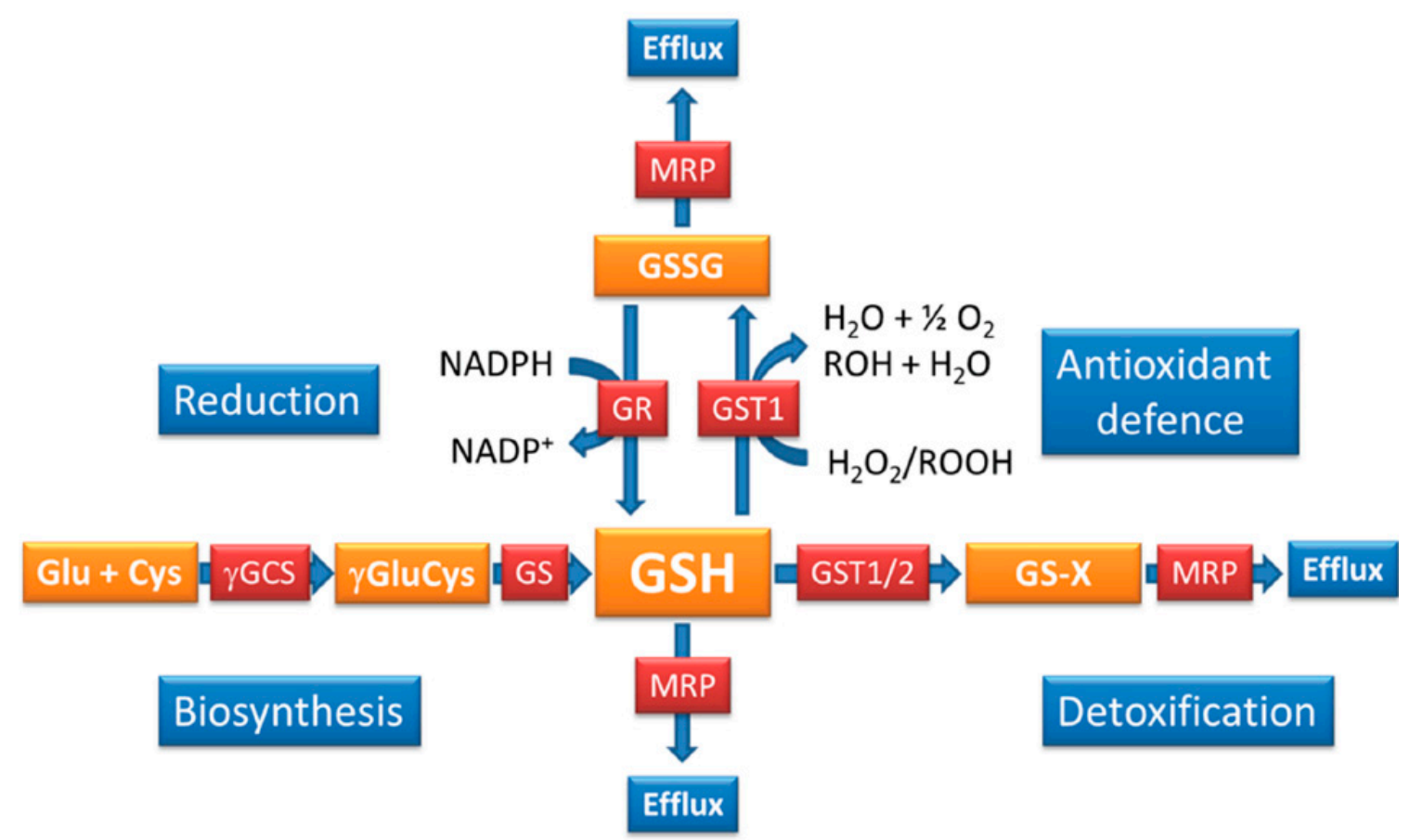

Figure 1. Regulation of GSH homeostasis in P. falciparum. Parasites contain GSH mainly in its reduced form [38] and its redox state is maintained by GR. GSSG and GSH are both effluxed from the parasites [46,49] to maintain GSH/GSSG redox balance. GSH is oxidised through antioxidant reactions primarily with GST1. Efflux, detoxification and antioxidant reactions lead to a constant loss of GSH, which is compensated for by GSH biosynthesis. Thus, efflux is counteracted by an active GSH biosynthesis, while oxidation of GSH is balanced through an active GR redox cycle.

\section{How is the GSH Redox Balance Regulated in Plasmodium?}

Important insights into the mechanisms that contribute to regulating GSH levels as well as its redox state were obtained through specific inhibition of de novo GSH biosynthesis or inhibition of GR in P. falciparum-infected RBC [41,44]. More precise and targeted manipulation of these two key reactions became possible through the knockout of genes encoding $\gamma$-GCS and GR, respectively, in $P$. berghei. This offered insights into the potential importance of these two proteins in the animal model malaria species and allows to some extent to conclude also about the regulatory roles of these reactions for the maintenance of GSH redox balance in the human malaria parasites (Figure 1 and Table 1) $[66,67,72]$. 
Table 1. Comparison of $P$. falciparum and $P$. berghei protein and metabolite characteristics involved in glutathione metabolism.

\begin{tabular}{|c|c|c|}
\hline & P. falciparum & P. berghei \\
\hline Protein/Gene ID & Characteristics & Characteristics \\
\hline \multirow{3}{*}{$\begin{array}{l}\text { Glutathione reductase (GR) } \\
\text { Pf3D7_1419800.1; PBANKA_102340 }\end{array}$} & Genetic validation: not done & Genetic validation: essential for sexual development [72] \\
\hline & Chemical validation: essential in RBC stages [73] & Chemical validation: not conclusive \\
\hline & Location: cytoplasm and apicoplast [39] & Location: not analysed \\
\hline \multirow{4}{*}{$\begin{array}{l}\text { Thioredoxin reductase (TrxR) } \\
\text { Pf3D7_0923800.1; PBANKA_082470 }\end{array}$} & Genetic validation: only possible in presence of & Genetic validation: not essential in any life cycle stage \\
\hline & expression plasmid [74] & \\
\hline & Chemical validation: essential in RBC stages [75] & Chemical validation: \\
\hline & Location: cytoplasm and mitochondrion [39] & Location: not investigated \\
\hline Glutathione S-transferase 1 (GST1) & Genetic validation: not done & Genetic validation: not done \\
\hline \multirow[t]{4}{*}{ Pf3D7_1419300; PBANKA_102390 } & Chemical validation: essential in RBC stages [76] & Chemical validation: not conclusive $[76,77]$ \\
\hline & Location: predicted to be cytoplasmic & Location: not analysed \\
\hline & No increase of activity with drug resistance [56] & Increased activity correlates with drug resistance [78-80] \\
\hline & Has antioxidant activity [56] & Not assessed \\
\hline Glutathione S-transferase 2 (EXP1) & Location: parasitophorous vacuolar membrane [54] & Protein was not studied with respect to GSH metabolism \\
\hline \multirow[t]{3}{*}{ Pf3D7_1121600; PBANKA_092670 } & Involved in heme and drug detoxification & \\
\hline & Antioxidant activity not determined & \\
\hline & Essentiality not determined & \\
\hline$\gamma$-Glutamylcysteine synthetase $(\gamma \mathrm{GCS})$ & Genetic validation: impossible to KO gene [44] & Genetic validation: essential for sexual development [67] \\
\hline \multirow[t]{3}{*}{ Pf3D7_0918900; PBANKA_081980 } & Chemical validation: specific inhibition with $\mathrm{BSO}$ is & Chemical validation: specific inhibition does not affect \\
\hline & lethal in RBC stages & parasite viability—not essential in RBC stages \\
\hline & Location: cytoplasm & Location: not studied \\
\hline \multirow[t]{2}{*}{ GSH and drug resistance } & GSH levels elevated in some drug resistant parasites $[41,81]$ & GSH levels elevated in drug resistant parasites $[78,79]$ \\
\hline & $\begin{array}{l}\text { GSH levels reduced in isogenic drug resistant } \\
\text { parasites }[45]\end{array}$ & \\
\hline
\end{tabular}


$P$. falciparum GR has been extensively studied over the past decades [20,82]. The gene was cloned, the recombinant protein expressed, characterised biochemically and its crystal structure was solved [35,83-86]. These studies allowed insights into the structural requirements of potential parasite-specific inhibitors and this has been taken forward in many studies since [73]. However, the recent gene deletion studies in $P$. berghei, a mouse malaria species that is frequently used as animal model for the human malaria species $P$. falciparum, revealed that GR is not essential for the survival of the intra-erythrocytic stages of $P$. berghei $[66,72]$. The knockout of $P$. berghei gr had little effect on parasite GSH levels and did not obviously affect intra-erythrocytic viability or parasite growth, providing strong evidence that GR in $P$. berghei is a redundant protein during intra-erythrocytic life and that its function is compensated for by alternative mechanisms, one being the thioredoxin redox system. This is reminiscent of Saccharamyces cerevisiae lacking $g r$, where the thioredoxin system provides an alternative reducing system that maintains the intracellular GSH/GSSG redox balance [87]. Apart from an alternative reducing system for GSSG, it is possible that the GSH/GSSG balance is sustained by efflux of GSSG and an increase of GSH de novo biosynthesis compensates for the loss of the tripeptide (Figure 1) $[40,44,49]$. This is feasible given that attempts to generate a double knockout mutant lacking both $g r$ and $\gamma$-gcs were unsuccessful in $P$. berghei [72]. Another possible mechanism that could compensate for the loss of GR function is the uptake of GSH, but there is little evidence that GSH uptake into the parasite is sufficiently efficient to allow for the loss of the highly efficient GR activity; although this has not been experimental assessed in $P$. berghei gr deletion mutants, which would be highly informative $[44,48]$. The data suggest that it is difficult to deregulate the GSSG/GSH redox balance efficiently during intra-erythrocytic growth of the parasites, particularly in $P$. berghei. This may not be the case in $P$. falciparum, where so far no report about a deletion of the $g r$ gene or the attempt thereof has been published (Table 1); in our lab a knockout of $P f g r$ was impossible to date (unpublished data). This implies that $P f G R$ may have a more pronounced function for the maintenance of the parasite's redox homeostasis during their intra-erythrocytic growth than is the case in P. berghei.

The deletion of $g r$ in $P$. berghei impacted however, on the progression of the parasites through their sexual cycle in the mosquito vector $[66,72]$. This part of the growth cycle was completely abrogated providing evidence that the loss of GR function in $P$. berghei cannot be replaced by the thioredoxin redox cycle during sexual development and that the two redox systems must have distinct roles during the parasites' progression through these stages of their life cycle. This is further emphasised by the finding that thioredoxin reductase is not essential in any of the life cycle stages (including the sexual stages) of the $P$. berghei [72], showing that although there is some functional redundancy between the two redox systems during the erythrocytic cycle their individual roles must differ significantly in other parts of the parasite's life cycle (Table 1). The precise reason for this selective effect of the $g r$ deletion is unknown, but it has been speculated that GSH plays a pivotal role during the development of the Plasmodium in the mosquito, because the mosquito itself does not contain GR but relies on the reduction of GSSG by alternative redox systems [88]. Interestingly, a gene disruption or deletion of thioredoxin reductase in the human malaria species $P$. falciparum was impossible potentially highlighting differences in the redox biology of different Plasmodium species (Table 1) [74]. However, it cannot be excluded that the unsuccessful attempts to generate a thioredoxin reductase deletion in $P$. falciparum reflect technical limitations of the $P$. falciparum in vitro culture and knockout techniques used at the time of the study. One way of circumventing these limitations is the recent 
advent of conditional gene deletion systems in P. falciparum [89], which will allow revisiting this approach and elucidating to what extend the requirement for active redox systems during intra-erythrocytic development of $P$. falciparum and $P$. berghei differ and may represent distinct and parasite-specific metabolic adaptations.

Apart from redundancy between the two major redox systems involving GR and thioredoxin reductase, GSH levels are regulated through the biosynthetic pathway (Figure 1). GSH is synthesized de novo by two consecutive ATP-dependent enzymatic reactions catalysed by $\gamma$-glutamylcysteine synthetase ( $\gamma$ GCS) and glutathione synthetase (GS) [32]. Plasmodium parasites possess both $\gamma$ GCS and GS [42,43]; both proteins are located in the parasite cytoplasm [45] similar to the situation in mammalian cells [90]. $\gamma \mathrm{GCS}$ is considered the rate-limiting step of GSH biosynthesis while GS appears to be constitutively active without being post-translationally regulated [32]. Availability of GSH either through de novo biosynthesis regulated by $\gamma \mathrm{GCS}$ or provided externally is essential in S. cerevisiae, Schizosaccharamyces pombe, Candida albicans as well as the protozoan parasite Trypanosoma brucei [91-96] and a knockout of $\gamma g c s$ in mammals results in embryonic lethality [97]. These data emphasise the importance of GSH biosynthetic pathway for maintaining an adequate level of GSH in multiple eukaryotes and suggests that it may be a suitable target for intervention strategies should this also hold true for the Plasmodium parasites.

Mammalian $\gamma \mathrm{GCS}$ has a catalytic and a regulatory subunit, which are distinct gene products Huang $[98,99]$. The enzymes from bacteria, fungi and the protozoan parasites T. brucei and Plasmodium consist only of the catalytic subunit [42,96,100-103], proposing that there are fundamental differences between the mammalian (host) proteins and those operating in protozoan parasites underpinning the potential for chemotherapeutic exploitation. In mammalian cells, the regulatory and catalytic subunits of $\gamma \mathrm{GCS}$ form a heterodimer, which increases catalytic activity and decreases response to GSH feedback inhibition $[104,105]$. The relative abundance of the two protein subunits differs in a tissue-specific manner allowing a site/tissue-specific regulation of GSH production [106].

Apart from the post-translational regulation of $\gamma \mathrm{GCS}$, GSH biosynthesis depends on the satisfactory supply of its precursor amino acids L-glutamate, L-cysteine and L-glycine with L-cysteine generally considered the limiting component [107]. The availability of the three amino acids links GSH biosynthesis directly to nitrogen, carbon and sulphur metabolism and thus reflects the cell's metabolic status. This is also the case in Plasmodium where metabolic re-modelling occurs many times in their developmental cycles in the two hosts. During the growth cycle in the RBC, the parasites obtain the majority of their amino acids through the digestion of hemoglobin. The only truly essential amino acid during this developmental stage of the parasites appears to be isoleucine while omitting other amino acids from the growth medium in vitro restricts growth but is not lethal to the parasites [108,109]. Genes encoding cystathionine $\beta$-synthase and cystathionine $\gamma$-lyase are lacking from the parasite genome [62] implying that they are unable to generate cysteine via transsulfuration from methionine and that they rely on cysteine/cystine uptake from their environment/host. Given that there is only a small number of cysteine residues (3) or methionine residues (5) present in the hemoglobin peptide chains restricting the availability of the sulphur-containing amino acid required for GSH biosynthesis, it is likely that an additional supply of sulphur-containing amino acids is required for optimal parasite growth. The availability of glutamine in vitro is high (the culture medium is usually supplemented with $2 \mathrm{mM}$ glutamine) and in vivo its serum concentration is $\sim 500 \mu \mathrm{M}$ [110]. Recent targeted metabolomics studies of Plasmodium carbon metabolism revealed that during blood stage development, the parasites 
rely heavily on the supply of exogenous glutamine delivering the carbon skeleton that is fed into the mitochondrial tricarboxylic acid cycle as well as providing amino nitrogen for transamination reactions [111,112]. The glycine concentration in human serum is $\sim 250 \mu \mathrm{M}$ and the amino acid may also be provided via carbon or lipid metabolism in the parasites, similar to other eukaryotes $[110,113,114]$. It is therefore likely that the parasites depend on a supply of the precursor amino acids required for GSH biosynthesis largely from their host and this may vary with their dietary situation. Studies investigating the importance of GSH biosynthesis in the human malaria parasite $P$. falciparum suggest that the pathway is important if not essential for parasite survival. This was established using both chemical and genetic approaches (Table 1) [40,44]. The deletion of the $\gamma g c s$ gene was impossible in $P$. falciparum suggesting that it is essential for the human malaria parasite and inhibition of GSH biosynthesis using the irreversible and specific inhibitor of $\gamma \mathrm{GCS}$ buthionine sulfoximine (BSO) killed the parasites at $\mu \mathrm{M}$ concentrations $[41,44]$. However, the biosynthesis of GSH is not essential for the intra-erythrocytic development of the murine malaria species P. berghei [67] and accordingly BSO has no discernible effect on these parasites even at high concentrations [115] (Table 1). The knockout of $\gamma g c s$ in $P$. berghei led to a reduction of total GSH levels and reduced growth rate of the mutant parasites in their mammalian host marginally. It was observed that mutant parasites preferably infected reticulocytes [67], which metabolically differ significantly from mature RBC [115].

The fact that a knockout of the $\gamma g c s$ gene was possible in $P$. berghei but not in the human malaria parasite $P$. falciparum is reminiscent of the data obtained for a gene deletion of $P$. falciparum and $P$. berghei thioredoxin reductase genes mentioned above (Table 1) [44,67]. Thioredoxin reductase was shown to be non-essential throughout the entire life cycle of $P$. berghei [72], while it was impossible to knockout the gene in P. falciparum [74]. These diverse results support the hypothesis that the host environment (for instance the parasite's host cell preference) may change and impact on parasite growth and survival rates and that therefore it is important to interrogate data from animal models carefully before drawing final conclusions as to the suitability of biological targets only validated in an animal model parasite. As outlined above, new conditional knockout systems are now becoming available to allow more robust assessment of drug targets in the human parasite $P$. falciparum experimentally. However, there are also important advantages of using the animal model parasite $P$. berghei to assess and validate potential drug target candidates in malaria parasites. The speed, ease and throughput with which the $P$. berghei system allows genetic manipulation and assessment of gene essentiality provides an enormous benefit [116] and the additional bonus of being able to assess the effects of gene knockouts on the growth and development of all life cycles stages of the parasites that are much less amenable to study with $P$. falciparum cannot be underestimated. In fact, using $P$. berghei it was demonstrated that the increased metabolic activity in the mitochondrion of sexual stages of the parasites is one reason for the essential role of GSH [67].

Other approaches that allowed insights into the regulatory mechanisms governing GSH/GSSG redox balance were obtained using the chemical inhibition of $\gamma \mathrm{GCS}$ by BSO in P. falciparum-infected RBC. It was shown that $P$. falciparum-infected RBC turn over GSH much faster than the uninfected host cell [40]; this highlights the importance of the pathway for the parasites. This was further accentuated by the fact that inhibition of $\gamma \mathrm{GCS}$ is lethal for $P$. falciparum in vitro, supporting that GSH biosynthesis is essential for parasite survival during the RBC cycle [44]. Overexpression of the $\gamma g c s$ gene in P. falciparum affected GSH redox homeostasis and resulted in an adaptive decrease of GR 
protein levels and an increase in GSH/GSSG efflux. These data support the idea that the GSH redox balance is carefully guarded and adjusted by GR activity and GSH/GSSG efflux, if $\gamma \mathrm{GCS}$ activity is elevated and presumably also when it is reduced [44,46,49]. These findings summarise that reduction of GSSG, biosynthesis of GSH and efflux of the tripeptide are primarily responsible for the regulation of intracellular GSH homeostasis (Figure 1).

Another mechanism that also might be involved and has been suggested to compensate for the loss of GSH biosynthesis is uptake of the tripeptide as has been shown in yeast $[48,117]$. However, the nature of the transporter is not clear and there are no obvious homologues of high-affinity GSH transporters belonging to the family of oligopeptide transporters (OPT) found in yeast, plants and bacteria in the $P$. falciparum genome [118]. However, a recent study has suggested that the DV chloroquine resistance transporter PfCRT is involved in oligopeptide transport in P. falciparum and the transporter has also been implicated in the transport of GSH possibly providing the tripeptide ingested by the parasites into the DV to the parasites $[45,119,120]$; plant homologues of PfCRT located in the chloroplast membrane were identified and shown to efficiently transport GSH [121].

\section{Glutathione and Its Role in Drug Resistance}

Using the redox active probe hGrx-roGFP a recent study showed that chloroquine, artemisinin derivatives, methylene blue, redox cyclers and pro-oxidants as well as BSO affect GSH redox balance in P. falciparum directly [38]. It was also demonstrated that the two P. falciparum strains 3D7 and Dd2 contain differential GSH redox capacities to respond to extracellular pro-oxidant challenges suggesting that the multidrug resistant parasites Dd2 have either elevated GSH concentrations or an elevated ability to maintain their GSH redox balance more efficiently than the drug sensitive parasites 3D7 [38,41]. This is in agreement with previous work showing that different parasite isolates contain differing levels of GSH and that these differences may correlate to some extent with their drug resistance phenotypes [38,41,45]. This hypothesis was particularly closely investigated for the antimalarial chloroquine and drug sensitive with drug resistant parasites were compared. The primary determinant of chloroquine resistance is associated with mutations in the P. falciparum PfCRT [122]. It is generally accepted that the principal mode of chloroquine action relies on its accumulation in the parasite DV due to its basic character; in the acidic environment of the DV, chloroquine is protonated trapping the drug in the DV where it interferes with the biomineralisation of toxic heme into hemozoin $[123,124]$. The release of heme is thought to be lethal to the parasites presumably due to its toxicity $[125,126]$. Mutations of PfCRT are associated with reduced accumulation of the drug in the DV and it was shown that the mutated transporter is responsible for the export of chloroquine from the DV. This was corroborated by in vitro studies showing that mutated PfCRT facilitates transport of chloroquine [45,119]. However, levels of chloroquine resistance vary considerably between parasite isolates suggesting other secondary determining or modulating factors being involved in the mechanism of chloroquine resistance. Mutations in PfPgh1 transporter, a member of the MDR family of ATP transporters, and PfMRP, a member of the multidrug resistance associated protein family, were shown to be secondary determinants of the level of chloroquine resistance [48,127-130] although the suggested correlation with mutations in PfMRP and the transporter's contribution to resistance against chloroquine is controversial $[48,131]$. 
In addition, varying concentrations of GSH were proposed to modulate the level of chloroquine resistance $[41,45,48,49,52]$. The association between elevated GSH levels and chloroquine resistance was convincingly shown in the animal model malaria species $P$. berghei. Increased GSH levels correlated with elevated GST activity, and reduced formation of hemozoin (presumably a higher detoxification of heme through the GSH-GST detoxification system) in the murine parasites (Table 1) $[47,78,79,132]$. For the human malaria parasite $P$. falciparum controversial data have been reported [45,133], which may reflect varying responses by the human malaria parasites to the changing capability of heme detoxification due to chloroquine action. However, upon treatment with chloroquine increased levels of hemin were found to accumulate in membranes isolated from $P$. falciparum-parasitized RBC, which correlated with parasite death [126] suggesting that formation of hemozoin is likely to be negatively affected upon chloroquine treatment. The hypothesis that elevated GSH levels increase resistance to chloroquine [41,126,134] was queried when the effect of GSH levels on chloroquine resistance was investigated in isogenic chloroquine sensitive and resistant $P$. falciparum. The study by Patzewitz and colleagues [45] showed that chloroquine resistant parasites (that only differ in their crt loci from the sensitive lines) had reduced levels of GSH rather than elevated GSH levels (Table 1) [45]. It was further demonstrated that mutant PfCRT transports GSH likely into the DV where it may have a role in protecting parasites against the toxicity of free heme by degrading it, leading to an increased tolerance towards the drug [45,81,135-138]. Support for this theory was recently provided by a genetic metabolomics/quantitative trait locus analysis evaluating the metabolite composition of the P. falciparum progeny of a genetic cross of chloroquine resistant and chloroquine sensitive parasites [120]. This study provided evidence that the natural role of PfCRT is linked to protein degradation in the DV and likely peptide transport across the DV membrane. It showed that mutations of PfCRT led to peptide accumulation (presumably due to reduced transport from the DV into the parasite cytoplasm), elevated levels of resistance against chloroquine (chloroquine possibly affecting peptide transport from the DV by competing for their transport by mutated PfCRT) and reduced fitness of the resistant parasites. The latter may be attributable to the reduced availability of peptides/amino acids for downstream metabolic and biosynthetic activities (including amino acids required for the synthesis of GSH) in the chloroquine resistant parasites [120].

Comparing parasite isolates/strains with different genetic backgrounds suggested that elevated GSH levels may provide protection against other antimalarials such as artemisinin and its derivatives [38]. The association of GSH may potentially be a contributing factor to the levels of resistance against this class of antimalarials; the major determinants for artemisinin-resistance in $P$. falciparum are however, mutations in the conserved kelch protein K13 (PF3D7_1343700) encoded on chromosome 13 [139-141]. These polymorphisms now provide the first molecular markers to detect artemisinin resistance and this will be of vital importance to prevent further spread of resistance against these extremely efficient antimalarials. Apart from its structural similarity to kelch-domain containing proteins, nothing is known about the function of $P f \mathrm{~K} 13$, but it has been speculated that it is involved in protein-protein interactions and the parasite's redox signalling in analogy to kelch-like proteins such as Keap1 and Nrf2 in other organisms [142]. 


\section{Glutathione Metabolism of Plasmodium-A Drug Target?}

Interfering with Plasmodium GSH homeostasis may provide a means to interfere with parasite survival at different stages of their complex life cycle. The parasites maintain a highly reducing intracellular GSH/GSSG ratio [38] thanks to GR and presumably their ability to modulate GSH and GSSG levels through efflux pumps [46,48,49]. GR has been studied extensively as potential target for drug discovery and 1,4-naphthoquinones and other redox cyclers have been identified as potential chemical leads [73]. Based on structural features of the parasite protein, chemicals binding in the homodimer intersubunit cavity were also found to be promising drug leads inhibiting the recombinant enzyme with high efficiency as well as displaying antiparasitic activity in vitro [143]. One downside is however, that GR was shown to be not essential in the RBC stages of P. berghei and unfortunately there is no information available about its essentiality in $P$. falciparum in vitro. Given that other proteins involved in the maintenance of the human parasite's redox balance such as thioredoxin reductase and $\gamma$-GCS appear to be essential for the survival of RBC stages of $P$. falciparum while they are dispensable for the murine malaria parasites, suggests that there is further need to fully assess the role of GR in $P$. falciparum before concluding that this protein is not a suitable target for intervention against RBC stages of human malaria. However, even if GR is not essential during RBC development, the protein appears to be a possible transmission blocking drug target (Table 1). Similarly, the rate limiting enzyme of GSH biosynthesis, $\gamma$-GCS, was found to be essential during sexual development of $P$. berghei although mutant parasites had reduced GSH levels and preferably infected reticulocytes during blood stage cycle suggesting a greater requirement for a supply of the tripeptide from their host and implying an imbalance of GSH redox homeostasis [67]. However, similar to GR, a knockout of the gene encoding $\gamma$-GCS in P. falciparum was impossible and using BSO, the need for an active GSH biosynthesis pathway during intra-erythrocytic development of the human malaria species was demonstrated (Table 1) [40,44]. A downside is, however, that chemical validation with BSO may not be entirely specific. It was suggested in a previous study on T. brucei $\gamma$-GCS that the drug may have off target effects [95]. Overall, the distinct findings from the studies using $P$. berghei and $P$. falciparum may reflect metabolic differences of the two malaria species and the essentiality of GR or $\gamma$-GCS should be investigated using the technical advances that are currently being made with conditional knockout systems (Table 1). Both gene deletions resulted in an interruption of the sexual development of $P$. berghei strongly suggesting that the GSSG reduction and GSH biosynthesis pathways may be suitable targets for transmission blocking agents. This goal to block malaria transmission is imperative to achieve eradication of the disease [144]. However, further experimental evidence is required to fully validate and assess the importance of the proteins of the human malaria species to conclude that the glutathione pathway of Plasmodium is suitable for future drug discovery.

It also needs to be assessed and validated whether the proposed targets are druggable. There is some doubt about the suitability of the disulphide oxidoreductase GR as the enzyme was assessed exhaustively as a drug target using multiple chemical compound screening and medicinal chemistry approaches and, so far, the outcomes have been modest and have not resulted in chemical leads that are taken forward into the drug development pipeline $[20,73,82,145]$. BSO on the other hand has been used as experimental tool to study the importance of GSH biosynthesis in cancer and also to chemically validate GSH biosynthesis in protozoan parasites $[40,44,95,146]$ and the notion of reducing 
intracellular GSH levels in combination with other treatments has been considered a valid approach in anticancer chemotherapy. However, the inability to recombinantly express the Plasmodium $\gamma$-GCS and the uncertainty about its essential function in the human Plasmodium species has hindered progress to fully evaluating Plasmodium $\gamma \mathrm{GCS}$ as a drug target. Overall, it remains for more robust and conclusive evidence to be generated to convince and engage the pharmaceutical industry in a drug discovery project with GSH metabolism of malaria parasites as a target.

\section{Acknowledgments}

The research was supported by the European Community's Seventh Framework Programme (FP7/2007-2013) under grant agreement N 242095 for supporting this research. The funders had no role in study design, data collection and analysis, decision to publish, or preparation of the manuscript.

\section{Conflicts of Interest}

The author declares no conflict of interest.

\section{List of Abbreviations}

BSO: buthionine sulfoximine; PfCRT: Plasmodium falciparum chloroquine resistance transporter; DV: digestive vacuole; ER: endoplasmatic reticulum; EXP1: exported protein 1; $\gamma$-GCS: $\gamma$-glutamylcysteine synthetase; GSH: reduced glutathione; GSSG: glutathione disulphide; GPx: glutathione peroxidase; GST: glutathione $S$-transferase; GR: glutathione reductase; GS: glutathione synthetase; PPP: pentose phosphate shunt; RBC: red blood cells.

\section{References}

1. WHO. World Malaria Report 2014; WHO Global Malaria Programme: Geneva, Switzerland, 2014.

2. Haldane, J.B. Suggestions as to quantitative measurement of rates of evolution. Evolution 1949, $3,51-56$.

3. Giribaldi, G.; Ulliers, D.; Mannu, F.; Arese, P.; Turrini, F. Growth of Plasmodium falciparum induces stage-dependent haemichrome formation, oxidative aggregation of band 3 , membrane deposition of complement and antibodies, and phagocytosis of parasitized erythrocytes. Br. J. Haematol. 2001, 113, 492-499.

4. Akide-Ndunge, O.B.; Ayi, K.; Arese, P. The Haldane malaria hypothesis: facts, artifacts, and a prophecy. Redox Rep. 2003, 8, 311-316.

5. Arese, P.; Turrini, F.; Schwarzer, E. Band 3/complement-mediated recognition and removal of normally senescent and pathological human erythrocytes. Cell. Physiol. Biochem. 2005, 16, 133-146.

6. Taylor, S.M.; Cerami, C.; Fairhurst, R.M. Hemoglobinopathies: Slicing the Gordian knot of Plasmodium falciparum malaria pathogenesis. PLoS Pathog. 2013, 9, e1003327.

7. Turrini, F.; Giribaldi, G.; Carta, F.; Mannu, F.; Arese, P. Mechanisms of band 3 oxidation and clustering in the phagocytosis of Plasmodium falciparum-infected erythrocytes. Redox Rep. 2003, 8, 300-303. 
8. Pantaleo, A.; Giribaldi, G.; Mannu, F.; Arese, P.; Turrini, F. Naturally occurring anti-band 3 antibodies and red blood cell removal under physiological and pathological conditions. Autoimmun. Rev. 2008, 7, 457-62.

9. Uyoga, S.; Skorokhod, O.A.; Opiyo, M.; Orori, E.N.; Williams, T.N.; Arese, P.; Schwarzer, E. Transfer of 4-hydroxynonenal from parasitized to non-parasitized erythrocytes in rosettes. Proposed role in severe malaria anemia. Br. J. Haematol. 2012, 157, 116-124.

10. Hunt, N.; Stocker, R. Oxidative stress and the redox status of malaria infected erythrocytes. Blood Cells 1990, 16, 499-526.

11. Ginsburg, H.; Atamna, H. The redox status of malaria-infected erythrocytes: An overview with an emphasis on unresolved problems. Parasite 1994, 1, 5-13.

12. Schwarzer, E.; Kuehn, H.; Arese, P. The hidden faces of hemozoin and its dangerous midwives. Trends Parasitol. 2003, 19, 197-198.

13. Schwarzer, E.; Kuhn, H.; Valente, E.; Arese, P. Malaria-parasitized erythrocytes and hemozoin nonenzymatically generate large amounts of hydroxy fatty acids that inhibit monocyte functions. Blood 2003, 101, 722-728.

14. Winograd, E.; Prudhomme, J.G.; Sherman, I.W. Band 3 clustering promotes the exposure of neoantigens in Plasmodium falciparum-infected erythrocytes. Mol. Biochem. Parasitol. 2005, 142, 98-105.

15. Skorokhod, O.A.; Caione, L.; Marrocco, T.; Migliardi, G.; Barrera, V.; Arese, P.; Piacibello, W.; Schwarzer, E. Inhibition of erythropoiesis in malaria anemia: Role of hemozoin and hemozoin-generated 4-hydroxynonenal. Blood 2010, 116, 4328-4337.

16. Aguilar, R.; Marrocco, T.; Skorokhod, O.A.; Barbosa, A.; Nhabomba, A.; Manaca, M.N.; Guinovart, C.; Quinto, L.; Arese, P.; Alonso, P.L.; et al. Blood oxidative stress markers and Plasmodium falciparum malaria in non-immune African children. Br. J. Haematol. 2014, 164, 438-450.

17. Becker, K.; Tilley, L.; Vennerstrom, J.L.; Roberts, D.; Rogerson, S.; Ginsburg, H. Oxidative stress in malaria parasite-infected erythrocytes: Host-parasite interactions. Int. J. Parasitol. 2004, 34, 163-189.

18. Müller, S. Redox and antioxidant systems of the malaria parasite Plasmodium falciparum. Mol. Microbiol. 2004, 53, 1291-1305.

19. Becker, K.; Rahlfs, S.; Nickel, C.; Schirmer, R.H. Glutathione-Functions and metabolism in the malarial parasite Plasmodium falciparum. Biol. Chem. 2003, 384, 551-566.

20. Jortzik, E.; Becker, K. Thioredoxin and glutathione systems in Plasmodium falciparum. Int. J. Med. Microbiol. 2012, 302, 187-194.

21. Müller, S.; Liebau, E.; Walter, R.D.; Krauth-Siegel, R.L. Thiol-based redox metabolism of protozoan parasites. Trends Parasitol. 2003, 19, 320-328.

22. Bozdech, Z.; Ginsburg, H. Data mining of the transcriptome of Plasmodium falciparum: The pentose phosphate pathway and ancillary processes. Malar. J. 2005, 4, doi:10.1186/1475-2875-4-17.

23. Bozdech, Z.; Ginsburg, H. Antioxidant defense in Plasmodium falciparum-Data mining of the transcriptome. Malar. J. 2004, 3, doi:10.1186/1475-2875-3-23.

24. Preuss, J.; Jortzik, E.; Becker, K. Glucose-6-phosphate metabolism in Plasmodium falciparum. IUBMB Life 2012, 64, 603-611. 
25. Preuss, J.; Maloney, P.; Peddibhotla, S.; Hedrick, M.P.; Hershberger, P.; Gosalia, P.; Milewski, M.; Li, Y.L.; Sugarman, E.; Hood, B.; et al. Discovery of a Plasmodium falciparum glucose-6-phosphate dehydrogenase 6-phosphogluconolactonase inhibitor $(R, Z)-N$-((1-ethylpyrrolidin-2-yl)methyl)-2(2-fluorobenzylidene)-3-oxo-3,4-dihydro-2H-benzo[b][1,4]thiazine-6-carboxamide (ML276) that reduces parasite growth in vitro. J. Med. Chem. 2012, 55, 7262-7272.

26. Jortzik, E.; Mailu, B.M.; Preuss, J.; Fischer, M.; Bode, L.; Rahlfs, S.; Becker, K. Glucose-6-phosphate dehydrogenase-6-phosphogluconolactonase: A unique bifunctional enzyme from Plasmodium falciparum. Biochem. J. 2011, 436, 641-650.

27. Storm, J.; Perner, J.; Aparicio, I.; Patzewitz, E.M.; Olszewski, K.; Llinas, M.; Engel, P.C.; Muller, S. Plasmodium falciparum glutamate dehydrogenase a is dispensable and not a drug target during erythrocytic development. Malar. J. 2011, 10, doi:10.1186/1475-2875-10-193.

28. Zocher, K.; Fritz-Wolf, K.; Kehr, S.; Fischer, M.; Rahlfs, S.; Becker, K. Biochemical and structural characterization of Plasmodium falciparum glutamate dehydrogenase 2. Mol. Biochem. Parasitol. 2012, 183, 52-62.

29. Wrenger, C.; Müller, S. Isocitrate dehydrogenase of Plasmodium falciparum. Eur. J. Biochem. 2003, 270, 1775-1783.

30. Krauth-Siegel, R.L.; Muller, J.G.; Lottspeich, F.; Schirmer, R.H. Glutathione reductase and glutamate dehydrogenase of Plasmodium falciparum, the causative agent of tropical malaria. Eur. J. Biochem. 1996, 235, 345-350.

31. Hudson, D.A.; Gannon, S.A.; Thorpe, C. Oxidative protein folding: From thiol-disulfide exchange reactions to the redox poise of the endoplasmic reticulum. Free Radic. Biol. Med. 2015, 80, 171-182.

32. Meister, A.; Anderson, M.E. Glutathione. Annu. Rev. Biochem. 1983, 52, 711-760.

33. Mullineaux, P.M.; Rausch, T. Glutathione, photosynthesis and the redox regulation of stress-responsive gene expression. Photosynth. Res. 2005, 86, 459-474.

34. Deponte, M. Glutathione catalysis and the reaction mechanisms of glutathione-dependent enzymes. Biochim. Biophys. Acta 2013, 1830, 3217-3266.

35. Farber, P.M.; Becker, K.; Muller, S.; Schirmer, R.H.; Franklin, R.M. Molecular cloning and characterization of a putative glutathione reductase gene, the PfGR2 gene, from Plasmodium falciparum. Eur. J. Biochem. 1996, 239, 655-661.

36. Krauth- Siegel, R.L.; Lohrer, H.; Buecheler, U.S.; Schirmer, R.H. The Antioxidant Enzymes Glutathione Reductase and Trypanotione Reductase as Drug Targets; Taylor \& Francis: London, UK; New York, NY, USA; Philadelphia, PA, USA, 1991; pp. 493-505.

37. Farber, P.M.; Arscott, L.D.; Williams, C.H., Jr.; Becker, K.; Schirmer, R.H. Recombinant Plasmodium falciparum glutathione reductase is inhibited by the antimalarial dye methylene blue. FEBS Lett. 1998, 422, 311-314.

38. Kasozi, D.; Mohring, F.; Rahlfs, S.; Meyer, A.J.; Becker, K. Real-time imaging of the intracellular glutathione redox potential in the malaria parasite Plasmodium falciparum. PLoS Pathog. 2013, 9, e1003782.

39. Kehr, S.; Sturm, N.; Rahlfs, S.; Przyborski, J.M.; Becker, K. Compartmentation of redox metabolism in malaria parasites. PLoS Pathog. 2011, 6, e1001242. 
40. Lüersen, K.; Walter, R.D.; Müller, S. Plasmodium falciparum-infected red blood cells depend on a functional glutathione de novo synthesis attributable to an enhanced loss of glutathione. Biochem. $J$. 2000, 346, 545-552.

41. Meierjohann, S.; Walter, R.D.; Müller, S. Regulation of intracellular glutathione levels in erythrocytes infected with chloroquine-sensitive and chloroquine-resistant Plasmodium falciparum. Biochem. J. 2002, 368, 761-768.

42. Lüersen, K.; Walter, R.D.; Müller, S. The putative gamma-glutamylcysteine synthetase from Plasmodium falciparum contains large insertions and a variable tandem repeat. Mol. Biochem. Parasitol. 1999, 98, 131-142.

43. Meierjohann, S.; Walter, R.D.; Müller, S. Glutathione synthetase from Plasmodium falciparum. Biochem. J. 2002, 363, 833-838.

44. Patzewitz, E.M.; Wong, E.H.; Müller, S. Dissecting the role of glutathione biosynthesis in Plasmodium falciparum. Mol. Microbiol. 2012, 83, 304-318.

45. Patzewitz, E.M.; Salcedo-Sora, J.E.; Wong, E.H.; Sethia, S.; Stocks, P.A.; Maughan, S.C.; Murray, J.A.; Krishna, S.; Bray, P.G.; Ward, S.A.; et al. Glutathione transport: A new role for PfCRT in chloroquine resistance. Antioxid. Redox Signal. 2013, 19, 683-695.

46. Barrand, M.A.; Winterberg, M.; Ng, F.; Nguyen, M.; Kirk, K.; Hladky, S.B. Glutathione export from human erythrocytes and Plasmodium falciparum malaria parasites. Biochem. J. 2012, 448, 389-400.

47. Lehane, A.M.; McDevitt, C.A.; Kirk, K.; Fidock, D.A. Degrees of chloroquine resistance in Plasmodium-Is the redox system involved? Int. J. Parasitol. Drugs Drug Resist. 2012, 2, 47-57.

48. Raj, D.K.; Mu, J.; Jiang, H.; Kabat, J.; Singh, S.; Sullivan, M.; Fay, M.P.; McCutchan, T.F.; $\mathrm{Su}$, X.Z. Disruption of a Plasmodium falciparum multidrug resistance-associated protein (PfMRP) alters its fitness and transport of antimalarial drugs and glutathione. J. Biol. Chem. 2009, 284, 7687-7696.

49. Atamna, H.; Ginsburg, H. The malaria parasite supplies glutathione to its host cell-Investigation of glutathione transport and metabolism in human erythrocytes infected with Plasmodium falciparum. Eur. J. Biochem. 1997, 250, 670-679.

50. Goldberg, D.E. Hemoglobin degradation. Curr. Top. Microbiol. Immunol. 2005, 295, 275-291.

51. Sigala, P.A.; Goldberg, D.E. The peculiarities and paradoxes of Plasmodium heme metabolism. Annu. Rev. Microbiol. 2014, 68, 259-278.

52. Atamna, H.; Ginsburg, H. Heme degradation in the presence of glutathione. A proposed mechanism to account for the high levels of non-heme iron found in the membranes of hemoglobinopathic red blood cells. J. Biol. Chem. 1995, 270, 24876-24883.

53. Loria, P.; Miller, S.; Foley, M.; Tilley, L. Inhibition of the peroxidative degradation of haem as the basis of action of chloroquine and other quinoline antimalarials. Biochem. J. 1999, 339, 363-370.

54. Lisewski, A.M.; Quiros, J.P.; Ng, C.L.; Adikesavan, A.K.; Miura, K.; Putluri, N.; Eastman, R.T.; Scanfeld, D.; Regenbogen, S.J.; Altenhofen, L.; et al. Supergenomic network compression and the discovery of EXP1 as a glutathione transferase inhibited by artesunate. Cell 2014, 158, 916-928. 
55. Klokouzas, A.; Tiffert, T.; van Schalkwyk, D.; Wu, C.P.; van Veen, H.W.; Barrand, M.A.; Hladky, S.B. Plasmodium falciparum expresses a multidrug resistance-associated protein. Biochem. Biophys. Res. Commun. 2004, 321, 197-201.

56. Harwaldt, P.; Rahlfs, S.; Becker, K. Glutathione S-transferase of the malarial parasite Plasmodium falciparum: Characterization of a potential drug target. Biol. Chem. 2002, 383, 821-830.

57. Fritz-Wolf, K.; Becker, A.; Rahlfs, S.; Harwaldt, P.; Schirmer, R.H.; Kabsch, W.; Becker, K. $\mathrm{X}$-ray structure of glutathione S-transferase from the malarial parasite Plasmodium falciparum. Proc. Natl. Acad. Sci. USA 2003, 100, 13821-13826.

58. Liebau, E.; Bergmann, B.; Campbell, A.M.; Teesdale-Spittle, P.; Brophy, P.M.; Luersen, K.; Walter, R.D. The glutathione S-transferase from Plasmodium falciparum. Mol. Biochem. Parasitol. 2002, 124, 85-90.

59. Perbandt, M.; Burmeister, C.; Walter, R.D.; Betzel, C.; Liebau, E. Native and inhibited structure of a $\mathrm{Mu}$ class-related glutathione S-transferase from Plasmodium falciparum. J. Biol. Chem. 2004, 279, 1336-1342.

60. Deponte, M.; Becker, K. Glutathione S-transferase from malarial parasites: Structural and functional aspects. Methods Enzymol. 2005, 401, 241-253.

61. Sztajer, H.; Gamain, B.; Aumann, K.D.; Slomianny, C.; Becker, K.; Brigelius-Flohe, R.; Flohe, L. The putative glutathione peroxidase gene of Plasmodium falciparum codes for a thioredoxin peroxidase. J. Biol. Chem. 2001, 276, 7397-7403.

62. Gardner, M.J.; Hall, N.; Fung, E.; White, O.; Berriman, M.; Hyman, R.W.; Carlton, J.M.; Pain, A.; Nelson, K.E.; Bowman, S.; et al. Genome sequence of the human malaria parasite Plasmodium falciparum. Nature 2002, 419, 498-511.

63. Kawazu, S.I.; Komaki-Yasuda, K.; Oku, H.; Kano, S. Peroxiredoxins in malaria parasites: Parasitologic aspects. Parasitol. Int. 2008, 57, 1-7.

64. Deponte, M.; Rahlfs, S.; Becker, K. Peroxiredoxin systems of protozoal parasites. SubCell. Biochem. 2007, 44, 219-229.

65. Ayi, K.; Cappadoro, M.; Branca, M.; Turrini, F.; Arese, P. Plasmodium falciparum glutathione metabolism and growth are independent of glutathione system of host erythrocyte. FEBS Lett. 1998, 424, 257-261.

66. Pastrana-Mena, R.; Dinglasan, R.R.; Franke-Fayard, B.; Vega-Rodriguez, J.; Fuentes-Caraballo, M.; Baerga-Ortiz, A.; Coppens, I.; Jacobs-Lorena, M.; Janse, C.J.; Serrano, A.E. Glutathione reductase-null malaria parasites have normal blood stage growth but arrest during development in the mosquito. J. Biol. Chem. 2010, 285, 27045-27056.

67. Vega-Rodriguez, J.; Franke-Fayard, B.; Dinglasan, R.R.; Janse, C.J.; Pastrana-Mena, R.; Waters, A.P.; Coppens, I.; Rodriguez-Orengo, J.F.; Srinivasan, P.; Jacobs-Lorena, M.; et al. The glutathione biosynthetic pathway of Plasmodium is essential for mosquito transmission. PLoS Pathog. 2009, 5, e1000302.

68. Tietze, F. Enzymic method for quantitative determination of nanogram amounts of total and oxidized glutathione: Applications to mammalian blood and other tissues. Anal. Biochem. 1969, 27, 502-522.

69. Urscher, M.; Alisch, R.; Deponte, M. The glyoxalase system of malaria parasites-Implications for cell biology and general glyoxalase research. Semin. Cell Dev. Biol. 2011, 22, 262-270. 
70. Deponte, M.; Becker, K.; Rahlfs, S. Plasmodium falciparum glutaredoxin-like proteins. Biol. Chem. 2005, 386, 33-40.

71. Kehr, S.; Jortzik, E.; Delahunty, C.; Yates, J.R., III; Rahlfs, S.; Becker, K. Protein S-glutathionylation in malaria parasites. Antioxid. Redox Signal. 2011, 15, 2855-2865.

72. Buchholz, K.; Putrianti, E.D.; Rahlfs, S.; Schirmer, R.H.; Becker, K.; Matuschewski, K. Molecular genetics evidence for the in vivo roles of the two major NADPH-dependent disulfide reductases in the malaria parasite. J. Biol. Chem. 2010, 285, 37388-37395.

73. Belorgey, D.; Lanfranchi, D.A.; Davioud-Charvet, E. 1,4-naphthoquinones and other NADPH-dependent glutathione reductase-catalyzed redox cyclers as antimalarial agents. Curr. Pharm. Des. 2013, 19, 2512-2528.

74. Krnajski, Z.; Gilberger, T.W.; Walter, R.D.; Cowman, A.F.; Müller, S. Thioredoxin reductase is essential for the survival of Plasmodium falciparum erythrocytic stages. J. Biol. Chem. 2002, 277, 25970-25975.

75. Andricopulo, A.D.; Akoachere, M.B.; Krogh, R.; Nickel, C.; McLeish, M.J.; Kenyon, G.L.; Arscott, L.D.; Williams, C.H., Jr.; Davioud-Charvet, E.; Becker, K. Specific inhibitors of Plasmodium falciparum thioredoxin reductase as potential antimalarial agents. Bioorg. Med. Chem. Lett. 2006, 16, 2283-2292.

76. Sturm, N.; Hu, Y.; Zimmermann, H.; Fritz-Wolf, K.; Wittlin, S.; Rahlfs, S.; Becker, K. Compounds structurally related to ellagic acid show improved antiplasmodial activity. Antimicrob. Agents Chemother. 2009, 53, 622-630.

77. Jortzik, E.; Fritz-Wolf, K.; Sturm, N.; Hipp, M.; Rahlfs, S.; Becker, K. Redox regulation of Plasmodium falciparum ornithine delta-aminotransferase. J. Mol. Biol. 2010, 402, 445-459.

78. Dubois, V.L.; Platel, D.F.; Pauly, G.; Tribouley-Duret, J. Plasmodium berghei: Implication of intracellular glutathione and its related enzyme in chloroquine resistance in vivo. Exp. Parasitol. 1995, 81, 117-124.

79. Platel, D.F.; Mangou, F.; Tribouley-Duret, J. Role of glutathione in the detoxification of ferriprotoporphyrin IX in chloroquine resistant Plasmodium berghei. Mol. Biochem. Parasitol. 1999, 98, 215-223.

80. Srivastava, P.; Puri, S.K.; Kamboj, K.K.; Pandey, V.C. Glutathione-S-transferase activity in malarial parasites. Trop. Med. Int. Health 1999, 4, 251-254.

81. Ginsburg, H.; Famin, O.; Zhang, J.; Krugliak, M. Inhibition of glutathione-dependent degradation of heme by chloroquine and amodiaquine as a possible basis for their antimalarial mode of action. Biochem. Pharmacol. 1998, 56, 1305-1313.

82. Krauth-Siegel, R.L.; Bauer, H.; Schirmer, R.H. Dithiol proteins as guardians of the intracellular redox milieu in parasites: Old and new drug targets in trypanosomes and malaria-causing plasmodia. Angew. Chem. Int. Ed. Engl. 2005, 44, 690-715.

83. Müller, S.; Gilberger, T.W.; Farber, P.M.; Becker, K.; Schirmer, R.H.; Walter, R.D. Recombinant putative glutathione reductase of Plasmodium falciparum exhibits thioredoxin reductase activity. Mol. Biochem. Parasitol. 1996, 80, 215-219.

84. Gilberger, T.W.; Schirmer, R.H.; Walter, R.D.; Muller, S. Deletion of the parasite-specific insertions and mutation of the catalytic triad in glutathione reductase from chloroquine-sensitive Plasmodium falciparum 3D7. Mol. Biochem. Parasitol. 2000, 107, 169-179. 
85. Sarma, G.N.; Savvides, S.N.; Becker, K.; Schirmer, M.; Schirmer, R.H.; Karplus, P.A. Glutathione reductase of the malarial parasite Plasmodium falciparum: Crystal structure and inhibitor development. J. Mol. Biol. 2003, 328, 893-907.

86. Savvides, S.N.; Scheiwein, M.; Bohme, C.C.; Arteel, G.E.; Karplus, P.A.; Becker, K.; Schirmer, R.H. Crystal structure of the antioxidant enzyme glutathione reductase inactivated by peroxynitrite. J. Biol. Chem. 2002, 277, 2779-2784.

87. Tan, S.X.; Greetham, D.; Raeth, S.; Grant, C.M.; Dawes, I.W.; Perrone, G.G. The thioredoxinthioredoxin reductase system can function in vivo as an alternative system to reduce oxidized glutathione in Saccharomyces cerevisiae. J. Biol. Chem. 2010, 285, 6118-6126.

88. Kanzok, S.M.; Fechner, A.; Bauer, H.; Ulschmid, J.K.; Muller, H.M.; Botella-Munoz, J.; Schneuwly, S.; Schirmer, R.; Becker, K. Substitution of the thioredoxin system for glutathione reductase in Drosophila melanogaster. Science 2001, 291, 643-646.

89. Collins, C.R.; Das, S.; Wong, E.H.; Andenmatten, N.; Stallmach, R.; Hackett, F.; Herman, J.P.; Muller, S.; Meissner, M.; Blackman, M.J. Robust inducible Cre recombinase activity in the human malaria parasite Plasmodium falciparum enables efficient gene deletion within a single asexual erythrocytic growth cycle. Mol. Microbiol. 2013, 88, 687-701.

90. Dalle-Donne, I.; Rossi, R.; Colombo, G.; Giustarini, D.; Milzani, A. Protein S-glutathionylation: A regulatory device from bacteria to humans. Trends Biochem. Sci. 2009, 34, 85-96.

91. Wu, G.; Fang, Y.Z.; Yang, S.; Lupton, J.R.; Turner, N.D. Glutathione metabolism and its implications for health. J. Nutr. 2004, 134, 489-492.

92. Grant, C.M.; MacIver, F.H.; Dawes, I.W. Glutathione synthetase is dispensable for growth under both normal and oxidative stress conditions in the yeast Saccharomyces cerevisiae due to an accumulation of the dipeptide gamma-glutamylcysteine. Mol. Biol. Cell 1997, 8, 1699-1707.

93. Grant, C.M.; MacIver, F.H.; Dawes, I.W. Glutathione is an essential metabolite required for resistance to oxidative stress in the yeast Saccharomyces cerevisiae. Curr. Genet. 1996, 29, $511-515$.

94. Chaudhuri, B.; Ingavale, S.; Bachhawat, A.K. $a p d_{1}^{+}$, a gene required for red pigment formation in ade6 mutants of Schizosaccharomyces pombe, encodes an enzyme required for glutathione biosynthesis: A role for glutathione and a glutathione-conjugate pump. Genetics 1997, 145, 75-83.

95. Huynh, T.T.; Huynh, V.T.; Harmon, M.A.; Phillips, M.A., Gene knockdown of gamma-glutamylcysteine synthetase by RNAi in the parasitic protozoa Trypanosoma brucei demonstrates that it is an essential enzyme. J. Biol. Chem. 2003, 278, 39794-39800.

96. Baek, Y.U.; Kim, Y.R.; Yim, H.S.; Kang, S.O. Disruption of gamma-glutamylcysteine synthetase results in absolute glutathione auxotrophy and apoptosis in Candida albicans. FEBS Lett. 2004, $556,47-52$.

97. Shi, Z.Z.; Osei-Frimpong, J.; Kala, G.; Kala, S.V.; Barrios, R.J.; Habib, G.M.; Lukin, D.J.; Danney, C.M.; Matzuk, M.M.; Lieberman, M.W. Glutathione synthesis is essential for mouse development but not for cell growth in culture. Proc. Natl. Acad. Sci. USA 2000, 97, 5101-5106.

98. Huang, C.S.; Anderson, M.E.; Meister, A. Amino acid sequence and function of the light subunit of rat kidney gamma-glutamylcysteine synthetase. J. Biol. Chem. 1993, 268, 20578-20583. 
99. Huang, C.S.; Chang, L.S.; Anderson, M.E.; Meister, A. Catalytic and regulatory properties of the heavy subunit of rat kidney gamma-glutamylcysteine synthetase. J. Biol. Chem. 1993, 268, 19675-19680.

100. Coblenz, A.; Wolf, K. Gcs1, a gene encoding gamma-glutamylcysteine synthetase in the fission yeast Schizosaccharomyces pombe. Yeast 1995, 11, 1171-1177.

101. Lueder, D.V.; Phillips, M.A. Characterization of Trypanosoma brucei gamma-glutamylcysteine synthetase, an essential enzyme in the biosynthesis of trypanothione (diglutathionylspermidine). J. Biol. Chem. 1996, 271, 17485-17490.

102. Griffith, O.W.; Mulcahy, R.T. The enzymes of glutathione synthesis: Gamma-glutamylcysteine synthetase. Adv. Enzymol. Relat. Areas Mol. Biol. 1999, 73, 209-267.

103. Kelly, B.S.; Antholine, W.E.; Griffith, O.W. Escherichia coli gamma-glutamylcysteine synthetase. Two active site metal ions affect substrate and inhibitor binding. J. Biol. Chem. 2002, 277, 50-58.

104. Backos, D.S.; Franklin, C.C.; Reigan, P. The role of glutathione in brain tumor drug resistance. Biochem. Pharmacol. 2012, 83, 1005-1012.

105. Lu, S.C. Glutathione synthesis. Biochim. Biophys. Acta 2013, 1830, 3143-3153.

106. Chen, Y.; Shertzer, H.G.; Schneider, S.N.; Nebert, D.W.; Dalton, T.P. Glutamate cysteine ligase catalysis: Dependence on ATP and modifier subunit for regulation of tissue glutathione levels. J. Biol. Chem. 2005, 280, 33766-33774.

107. Liu, Y.; Hyde, A.S.; Simpson, M.A.; Barycki, J.J. Emerging regulatory paradigms in glutathione metabolism. Adv. Cancer Res. 2014, 122, 69-101.

108. Liu, J.; Istvan, E.S.; Gluzman, I.Y.; Gross, J.; Goldberg, D.E. Plasmodium falciparum ensures its amino acid supply with multiple acquisition pathways and redundant proteolytic enzyme systems. Proc. Natl. Acad. Sci. USA 2006, 103, 8840-8845.

109. Babbitt, S.E.; Altenhofen, L.; Cobbold, S.A.; Istvan, E.S.; Fennell, C.; Doerig, C.; Llinas, M.; Goldberg, D.E. Plasmodium falciparum responds to amino acid starvation by entering into a hibernatory state. Proc. Natl. Acad. Sci. USA 2012, 109, E3278-E3287.

110. Psychogios, N.; Hau, D.D.; Peng, J.; Guo, A.C.; Mandal, R.; Bouatra, S.; Sinelnikov, I.; Krishnamurthy, R.; Eisner, R.; Gautam, B.; et al. The human serum metabolome. PLoS ONE 2011, 6, e16957.

111. MacRae, J.I.; Dixon, M.W.; Dearnley, M.K.; Chua, H.H.; Chambers, J.M.; Kenny, S.; Bottova, I.; Tilley, L.; McConville, M.J. Mitochondrial metabolism of sexual and asexual blood stages of the malaria parasite Plasmodium falciparum. BMC Biol. 2013, 11, 67, doi:10.1186/1741-7007-11-67.

112. Storm, J.; Sethia, S.; Blackburn, G.J.; Chokkathukalam, A.; Watson, D.G.; Breitling, R.; Coombs, G.H.; Muller, S. Phosphoenolpyruvate carboxylase identified as a key enzyme in erythrocytic Plasmodium falciparum carbon metabolism. PLoS Pathog. 2014, 10, e1003876.

113. Jain, R.; Adhikary, H.; Jha, S.; Jha, A.; Kumar, G.N. Remodulation of central carbon metabolic pathway in response to arsenite exposure in Rhodococcus sp. strain NAU-1. Microb. Biotechnol. 2012, 5, 764-772.

114. Locasale, J.W. Serine, glycine and one-carbon units: Cancer metabolism in full circle. Nat. Rev. Cancer 2013, 13, 572-583. 
115. Srivastava, A.; Creek, D.J.; Evans, K.J.; de Souza, D.; Schofield, L.; Muller, S.; Barrett, M.P.; McConville, M.J.; Waters, A.P. Host Reticulocytes Provide Metabolic Reservoirs That Can Be Exploited by Malaria Parasites. PLoS Pathog 2015, 11, e1004882.

116. Gomes, A.R.; Bushell, E.; Schwach, F.; Girling, G.; Anar, B.; Quail, M.A.; Herd, C.; Pfander, C.; Modrzynska, K.; Rayner, J.C.; et al. A genome-scale vector resource enables high-throughput reverse genetic screening in a malaria parasite. Cell Host Microbe 2015, 17, 404-413.

117. Bourbouloux, A.; Shahi, P.; Chakladar, A.; Delrot, S.; Bachhawat, A.K. Hgt1p, a high affinity glutathione transporter from the yeast Saccharomyces cerevisiae. J. Biol. Chem. 2000, 275, 13259-13265.

118. Bachhawat, A.K.; Thakur, A.; Kaur, J.; Zulkifli, M. Glutathione transporters. Biochim. Biophys. Acta 2013, 1830, 3154-3164.

119. Martin, R.E.; Marchetti, R.V.; Cowan, A.I.; Howitt, S.M.; Broer, S.; Kirk, K. Chloroquine transport via the malaria parasite's chloroquine resistance transporter. Science 2009, 325, 1680-1682.

120. Lewis, I.A.; Wacker, M.; Olszewski, K.L.; Cobbold, S.A.; Baska, K.S.; Tan, A.; Ferdig, M.T.; Llinas, M. Metabolic QTL analysis links chloroquine resistance in Plasmodium falciparum to impaired hemoglobin catabolism. PLoS Genet. 2014, 10, e1004085.

121. Maughan, S.C.; Pasternak, M.; Cairns, N.; Kiddle, G.; Brach, T.; Jarvis, R.; Haas, F.; Nieuwland, J.; Lim, B.; Muller, C.; et al. Plant homologs of the Plasmodium falciparum chloroquine-resistance transporter, PfCRT, are required for glutathione homeostasis and stress responses. Proc. Natl. Acad. Sci. USA 2010, 107, 2331-2336.

122. Fidock, D.A.; Nomura, T.; Talley, A.K.; Cooper, R.A.; Dzekunov, S.M.; Ferdig, M.T.; Ursos, L.M.; Sidhu, A.B.; Naude, B.; Deitsch, K.W.; et al. Mutations in the P. falciparum digestive vacuole transmembrane protein PfCRT and evidence for their role in chloroquine resistance. Mol. Cell 2000, 6, 861-871.

123. Bray, P.G.; Martin, R.E.; Tilley, L.; Ward, S.A.; Kirk, K.; Fidock, D.A. Defining the role of PfCRT in Plasmodium falciparum chloroquine resistance. Mol. Microbiol. 2005, 56, 323-333.

124. Lakshmanan, V.; Bray, P.G.; Verdier-Pinard, D.; Johnson, D.J.; Horrocks, P.; Muhle, R.A.; Alakpa, G.E.; Hughes, R.H.; Ward, S.A.; Krogstad, D.J.; et al. A critical role for PfCRT K76T in Plasmodium falciparum verapamil-reversible chloroquine resistance. EMBO J. 2005, 24, 2294-2305.

125. Kumar, S.; Bandyopadhyay, U. Free heme toxicity and its detoxification systems in human. Toxicol. Lett. 2005, 157, 175-188.

126. Famin, O.; Krugliak, M.; Ginsburg, H. Kinetics of inhibition of glutathione-mediated degradation of ferriprotoporphyrin IX by antimalarial drugs. Biochem. Pharmacol. 1999, 58, 59-68.

127. Reed, M.B.; Saliba, K.J.; Caruana, S.R.; Kirk, K.; Cowman, A.F. Pgh1 modulates sensitivity and resistance to multiple antimalarials in Plasmodium falciparum. Nature 2000, 403, 906-909.

128. Duraisingh, M.T.; Cowman, A.F. Contribution of the pfmdr1 gene to antimalarial drug-resistance. Acta Trop. 2005, 94, 181-190.

129. Sanchez, C.P.; Dave, A.; Stein, W.D.; Lanzer, M. Transporters as mediators of drug resistance in Plasmodium falciparum. Int. J. Parasitol. 2010, 40, 1109-1118.

130. Mu, J.; Ferdig, M.T.; Feng, X.; Joy, D.A.; Duan, J.; Furuya, T.; Subramanian, G.; Aravind, L.; Cooper, R.A.; Wootton, J.C.; et al. Multiple transporters associated with malaria parasite responses to chloroquine and quinine. Mol. Microbiol. 2003, 49, 977-989. 
131. Anderson, T.J.; Nair, S.; Qin, H.; Singlam, S.; Brockman, A.; Paiphun, L.; Nosten, F. Are transporter genes other than the chloroquine resistance locus (pfcrt) and multidrug resistance gene (pfmdr) associated with antimalarial drug resistance? Antimicrob. Agents Chemother. 2005, 49, 2180-2188.

132. Deharo, E.; Barkan, D.; Krugliak, M.; Golenser, J.; Ginsburg, H. Potentiation of the antimalarial action of chloroquine in rodent malaria by drugs known to reduce cellular glutathione levels. Biochem. Pharmacol. 2003, 66, 809-817.

133. Gligorijevic, B.; Bennett, T.; McAllister, R.; Urbach, J.S.; Roepe, P.D. Spinning disk confocal microscopy of live, intraerythrocytic malarial parasites. 2. Altered vacuolar volume regulation in drug resistant malaria. Biochemistry 2006, 45, 12411-12423.

134. Ginsburg, H.; Golenser, J. Glutathione is involved in the antimalarial action of chloroquine and its modulation affects drug sensitivity of human and murine species of Plasmodium. Redox Rep. 2003, 8, 276-279.

135. Hang, H.; Paguio, M.; Roepe, P.D. The antimalarial drug resistance protein Plasmodium falciparum chloroquine resistance transporter binds chloroquine. Biochemistry 2004, 43, 8290-8296.

136. Zhang, J.; Krugliak, M.; Ginsburg, H. The fate of ferriprotorphyrin IX in malaria infected erythrocytes in conjunction with the mode of action of antimalarial drugs. Mol. Biochem. Parasitol. 1999, 99, 129-141.

137. Famin, O.; Ginsburg, H. The treatment of Plasmodium falciparum-infected erythrocytes with chloroquine leads to accumulation of ferriprotoporphyrin IX bound to particular parasite proteins and to the inhibition of the parasite's 6-phosphogluconate dehydrogenase. Parasite 2003, 10, 39-50.

138. Famin, O.; Ginsburg, H. Differential effects of 4-aminoquinoline-containing antimalarial drugs on hemoglobin digestion in Plasmodium falciparum-infected erythrocytes. Biochem. Pharmacol. 2002, 63, 393-398.

139. Ariey, F.; Witkowski, B.; Amaratunga, C.; Beghain, J.; Langlois, A.C.; Khim, N.; Kim, S.; Duru, V.; Bouchier, C.; Ma, L.; et al. A molecular marker of artemisinin-resistant Plasmodium falciparum malaria. Nature 2014, 505, 50-55.

140. Miotto, O.; Amato, R.; Ashley, E.A.; MacInnis, B.; Almagro-Garcia, J.; Amaratunga, C.; Lim, P.; Mead, D.; Oyola, S.O.; Dhorda, M.; et al. Genetic architecture of artemisinin-resistant Plasmodium falciparum. Nat. Genet. 2015, 47, 226-234.

141. Takala-Harrison, S.; Jacob, C.G.; Arze, C.; Cummings, M.P.; Silva, J.C.; Dondorp, A.M.; Fukuda, M.M.; Hien, T.T.; Mayxay, M.; Noedl, H.; et al. Independent emergence of artemisinin resistance mutations among Plasmodium falciparum in Southeast Asia. J. Infect. Dis. 2015, 211, $670-679$.

142. Uruno, A.; Yagishita, Y.; Yamamoto, M. The Keap1-Nrf2 system and diabetes mellitus. Arch. Biochem. Biophys. 2015, 566, 76-84.

143. Tyagi, C.; Bathke, J.; Goyal, S.; Fischer, M.; Dahse, H.M.; Chacko, S.; Becker, K.; Grover, A. Targeting the intersubunit cavity of Plasmodium falciparum glutathione reductase by a novel natural inhibitor: Computational and experimental evidence. Int. J. Biochem. Cell Biol. 2015, 61, 72-80.

144. Leroy, D.; Campo, B.; Ding, X.C.; Burrows, J.N.; Cherbuin, S. Defining the biology component of the drug discovery strategy for malaria eradication. Trends Parasitol. 2014, 30, 478-490. 
145. Jaeger, T.; Flohe, L. The thiol-based redox networks of pathogens: Unexploited targets in the search for new drugs. Biofactors 2006, 27, 109-120.

146. Estrela, J.M.; Ortega, A.; Obrador, E. Glutathione in cancer biology and therapy. Crit. Rev. Clin. Lab. Sci. 2006, 43, 143-181.

(C) 2015 by the authors; licensee MDPI, Basel, Switzerland. This article is an open access article distributed under the terms and conditions of the Creative Commons Attribution license (http://creativecommons.org/licenses/by/4.0/). 